\title{
Animal Model of Depression: Pharmacological Sensitivity of a Hedonic Deficit
}

\author{
RICHARD J. KATZ ${ }^{1}$ \\ Mental Health Research Institute, Department of Psychiatry, University of Michigan Medical Center \\ Ann Arbor, MI 48109
}

Received 16 January 1981

\begin{abstract}
KATZ, R. J. Animal model of depression: Pharmacological sensitivity of a hedonic deficit. PHARMAC. BIOCHEM. BEHAV. 16(6) 965-968, 1982.-A reduction in sucrose and saccharine consumption following chronic stress is reported for the rat. This deficit may be related to consummatory deficits seen in endogenous depression. To further examine this state pharmacologically, stressed rats were treated with the antidepressant imipramine. Despite a general absence of appetitive effects (or in some cases mild anorexia) imipramine significantly restored saccharine consumption in a variety of tests. The pharmacological similarity of the deficit to the changes accompanying affective disorders further supports the potential applicability of the chronic stress model.
\end{abstract}

Animal model of depression Antidepressant Appetite Imipramine Saccharine Sucrose

WE previously have demonstrated that chronic unpredictable stress produces a behavioral, motivational, and neuroendocrine syndrome in the rat which closely resembles endogenous depression in humans $[2,3,8]$. In preliminary studies we also have identified a pervasive appetitive disruption involving reduced consumption of both nutritive and nonnutritive substances. For example, both sucrose and saccharine consumption were curtailed by up to $50 \%$ [3]. Weight loss and appetitive disturbances are also common in severe depression $[4,5]$.

Although other aspects of the chronic stress syndrome were shown to be responsive to pharmacotherapy or somatic therapy the effect of drugs upon appetitive disruptions were not examined. However, it might be predicted from previous studies on other aspects of the stress syndrome that antidepressant drugs would be particularly beneficial in restoring otherwise reduced consumption. The first purpose of the present report was to provide a more rigorous and thorough demonstration of key aspects of the previously reported deficits in sucrose and saccharine consumption. In addition the tricyclic antidepressant imipramine was examined for its ability to restore saccharine consumption in the rat. Imipramine is a standard tricyclic antidepressant in wide clinical use and is not known to possess intrinsic appetite stimulatory effects, at least following systemic administration in the rat. Saccharine was chosen to allow extension of previous studies and minimize any problems due to direct nutritional effects of the consumed substance.
METHOD

Subjects

A total of 200 experimentally naive adult male Sprague Dawley rats ( $n=8 /$ cell for the first experiment; $6 /$ cell for the final 3 experiments) each 70 days at the start of testing were maintained with food (Teklad 4.0\% fat rodent diet S-0836) and tap water continuously available and $12 \mathrm{hr} / 12 \mathrm{hr}$ lighting cycles (lights on $=0700-1900$ )

\section{Behavioral Procedures}

Procedures are essentially similar to those reported by Katz [2,3]. Rats were subjected to three weeks of chronic intermittent stress. The following stress regimen was in effect: exposure to 60 minutes of unpredictable shock (average $1 \mathrm{~mA}, 1-10 \mathrm{sec}$ duration; average a shock/min (3 exposures)), $40 \mathrm{hr}$ food deprivation ( 2 times), cold swim at $4^{\circ} \mathrm{C}$ for five min ( 3 times), 40 hr water deprivation ( 2 times), 5 min exposure to the heat stress at $40^{\circ} \mathrm{C}$ ( 2 times), 30 minutes shaker stress ( 2 times), reversal of day/night cycle ( 2 times). Stressors were delivered in a semirandom fashion every 2-3 days throughout the lighting cycle from $0800 \mathrm{hr}$ through 2200 $\mathrm{hr}$. Order of stress administration was identical to previous descriptions and has been presented elsewhere $[1,3,8]$.

The first experiment involved acute $(14 \mathrm{hr})$ exposure to a $100 \mathrm{ml}$ drinking tube containing vehicle (distilled water) or one of the two following solutions: sodium saccharine $(0.000$,

\footnotetext{
'Send reprint requests to Dr. Katz at: Department of Psychology, The Johns Hopkins University, 34th and Charles Streets, Baltimore, MD 21218.
} 


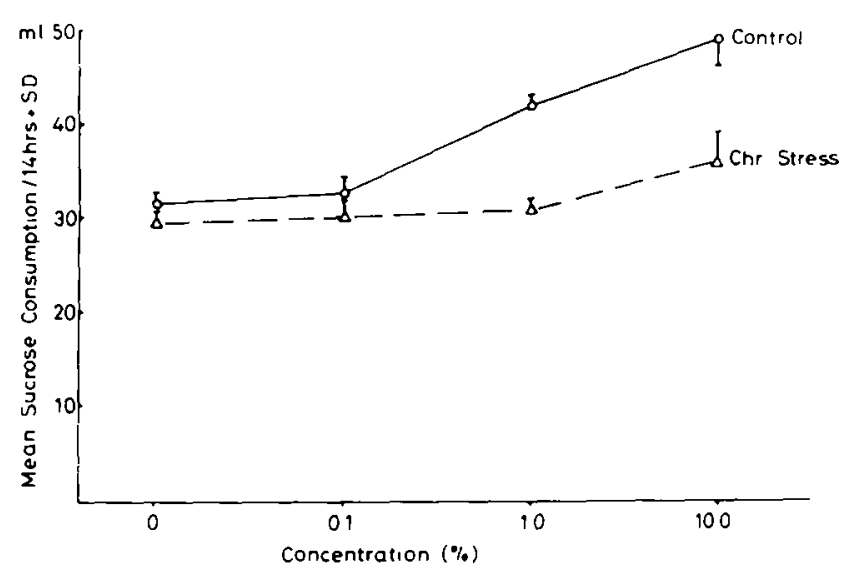

FIG. 1. Mean $\mathrm{ml}$ sucrose solution consumed $(+\mathrm{SD})$ by chronically stressed $(\triangle-\Delta)$ and unstressed control $(O-O)$ rats in a $14 \mathrm{hr}$ overnight exposure. A single test was carried out approximately 48 $\mathrm{hr}$ following the final stress of a 21 day stress period, or an equivalent period of undisturbed housing for controls. See text and Table 1 for details of stress procedure.

$0.0125,0.125$, and $1.25 \%)$; or sucrose $(0.0,0.1,1.0$, and $10.0 \%$ ). A 14 hour exposure beginning at $1800 \mathrm{hr}$ was employed to minimize initial neophobia. Exposure occurred 24 to $48 \mathrm{hr}$ after the final stressor to allow recovery from any acute effects of the stress regimen and comparison with previous data from our laboratory.

In a second set of experiments rats again were given a single $14 \mathrm{hr}$ exposure to a $100 \mathrm{ml}$ drinking tube containing distilled water or $0.125 \%$ commercial grade sodium saccharine with testing also beginning at $1800 \mathrm{hr}$. Unless specified to the contrary all rats in the second phase had a separate bottle of tap water. Three closely related studies were conducted using a $2 \times 2$ factorial design. In all cases the first factor was the presence or absence of chronic stress. For the first of these studies presence $v$ s absence of imipramine $\mathrm{HCl}$ $(5 \mathrm{mg} / \mathrm{kg} / \mathrm{day})$ represented the additional experimental factor. For the second study distilled water vehicle was used instead of sodium saccharine, and imipramine again was used as the therapeutic drug. A final study was carried out using the same design as experiment 2 , but normal tap water consumption from a single bottle was monitored. All drugs were given chronically (once daily for the three week stress regimen). Injections were intraperitoneal at $1 \mathrm{ml} / \mathrm{kg}$ of body weight. $0.9 \%$ Sodium chloride served as the imipramine vehicle.

All results are presented as means and standard deviations, and were analyzed using factorial analysis of variance. with Sheffé limits for post-hoc comparisons.

\section{RESULTS}

It should be noted at the outset that chronically stressed rats did not appear debilitated or incapable of responding at the time of testing. In previously published studies we have noted roughly equivalent basal open field performance in stressed and control rats $[3,4]$, although this does not preclude more subtle deficits.

Roughly equivalent consumption of vehicle occurred for all groups in the first study (Figs. 1,2) i.e., in no case did

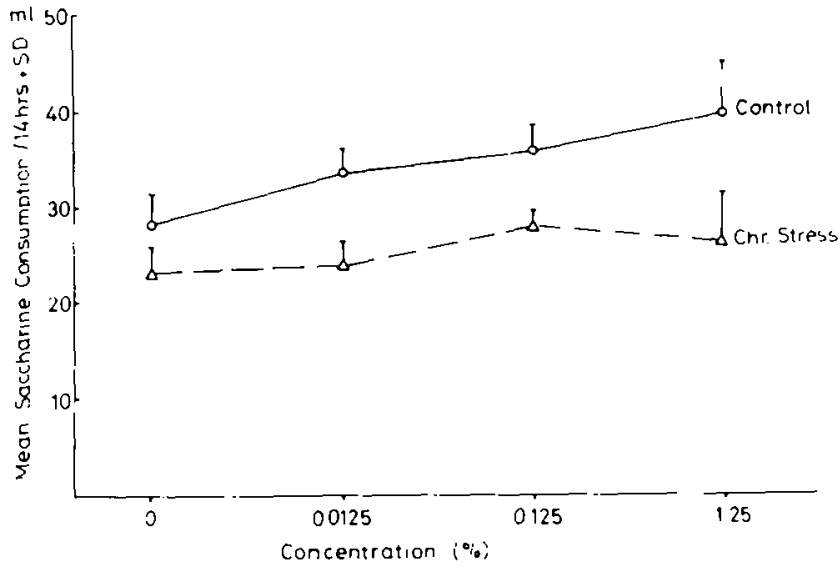

FIG. 2. Mean $\mathrm{ml}$ sodium saccharine solution consumed $(+\mathrm{SD})$ by chronically stressed $(\triangle-\triangle)$ and unstressed control $(C-O)$ rats in a $14 \mathrm{hr}$ overnight exposure. A single test was carried out approximately $48 \mathrm{hr}$ following the final stress of a 21 day stress period, or an equivalent period of undisturbed housing for controls. See text and Table 1 for details of stress procedure.

groups differ significantly when tested at $0 \%$ solutions (all is $<1$ ).

Specific consummatory deficits were identified for the chronically stressed rats based upon the additional presence of a nutritive (Fig. 1) or non-nutritive (Fig. 2) sweetener. These findings were confirmed statistically. In both cases. significant effects of groups were present across cells, $F(1,44)$ for sucrose and saccharine respectively $=28.4,13.1$, $p<0.001$. A significant effect of concentration was present for sucrose, $\mathrm{F}(3,44)=56.4, p<0.0001$, and marginally present for saccharine, $F(3,44)=2.2, p \sim 0.1$. Finally, no interaction effects were present, $F(3,44)=1.2,2.0$ respectively, $p>0.1$.

The findings from the final three studies indicate that a consummatory deficit again was present, and that it was selectively reversed by antidepressant treatment. Finally, the consummatory deficit was also seen in water drinking in at least certain circumstances. Findings are presented in Tables $1-3$ and below.

It may be seen in Table 1 that imipramine slightly depressed normal consumption in unstressed rats. Despite this, it significantly increased the level of consumption in chronically stressed animals. Both a stress effect, $\mathrm{F}(1,21)=39.7, p<0.0001$, and a stress by drug interaction. $F(1,21)=12.4, p<0.002$, were present, although a main effect for drug was not, $F=1.6$. The experimental hypothesis is supported by this last interaction.

Imipramine next was examined in a 2-bottle test using a distilled water vehicle rather than saccharine. It may be seen in Table 2 that no recovery was found. A chronic stress effect was present, $F(3,44)=51.7, p<0.00001$, however, neither a main effect for drugs, $F=0.8$, nor an interaction, $F=0.07$, were present to a statistically significant degree. It should be noted that significant reduction in water consumption by chronic stress is present in control rats, $\mathrm{F}=24, p<0.0001$.

Finally, Table 3 shows the effects of imipramine and stress upon normal tap water consumption in a single bottle test. It is evident that the deficits in a 1-bottle test using a typically consumed fluid are less. A stress effect is present, $F=6.3, p<0.05$, however, drug and interaction effects are not. $F=1.4,2.3$, respectively. 
TABLE 1

EFFECTS OF CHRONIC IMIPRAMINE HCI UPON SACCHARINF CONSUMPTION (IN ml) IN NORMAL AND

CHRONICALLY STRESSED RATS

\begin{tabular}{llc} 
& \multicolumn{2}{c}{ Drug } \\
Stress & Vehicle & Imipramine \\
Condition & $43.8 \pm 7.9$ & $29.3 \pm 10.3^{*+}$ \\
Control & $14.0 \pm 2.8^{*}$ & $20.8 \pm 6.6^{* \dagger}$ \\
Chronic & & \\
\hline
\end{tabular}

${ }^{*}$ Differs from control vehicle $p<0.05, \mathrm{~F} \cdot \mathrm{s}=48.3,11.4,18.7$.

†Differs from chronic stress vehicle $p<0.05$, F's $=12.8,4.2$. All F's left to right.

†Respective mean water consumption + sem in $\mathrm{ml}$ for the 4 groups $=21 \pm 8 ; 18 \pm 4 ; 15 \pm 6 ; 19 \pm 5 ;$ all values read left to right. ( $F$ for water consumption $=1.4$ n.s.).

\section{DISCUSSION}

From the initial findings it is apparent that the consumption deficit occurs both with a nutritive and a non-nutritive substance. Given a caloric differential between these substances this can not directly represent an alteration of caloric regulation. Whether the effect is present with other liquid diets, i.e., with milk or with solid diets remains to be determined, although clearly the present results would be consistent with changes in related designs. Insofar as common hedonically based properties may be adduced for sucrose and saccharine the deficit might be argued to be related to the hedonic disturbances of depression.

From the final three studies it is clear that the consummatory deficit is most pronounced in conjunction with a sweet solution, although apparently also present to some degree in other circumstances. No explanation of imipramine's effects upon basal consumption is apparent. We do note that imipramine was depressant and that this does not normally occur in clinical circumstances. It may be that this reflects anticholinergic factors or some interaction with neophobia. Alternately, chronic imipramine-administration may cause recurrent noradrenergic stimulation with subsequent adaptive changes at the cellular level. Were this the case, a smaller deficit after joint treatment might be due to nonadditive but physiologically similar effects. At present this must remain a matter of speculation. The findings partially confirm previous preliminary observations, and suggest for whichever of the above reasons that a specific reversal of the consummatory deficit associated with a sweet solution may be obtained through antidepressant treatment. These findings may bear a relation to weight loss during depression and weight gain during antidepressant treatment.

It must be noted that chronic stress effects in vehicle consuming groups were not found to a significant degree in the first study. Non-significant and quantitatively smaller deficits were observed, and indeed they are apparent to a more pronounced degree in the final studies, reaching a criterion of statistical significance. It might also be noted that a deficit in vehicle consumption has now been observed in 6 of 6 consecutive replications. This is likely to occur less than one time per hundred, and further argues for the presence of at least some vehicle related effect. It is perhaps not too
TABLE 2

EFFECTS OF CHRONIC IMIPRAMINE HCI UPON VEHICLF CONSUMPTION (IN ml) IN NORMAL AND CHRONICALLY STRESSED RATS

\begin{tabular}{lll} 
& \multicolumn{2}{c}{ Drug } \\
$\begin{array}{l}\text { Stress } \\
\text { Condition }\end{array}$ & Vehicle & Imipramine \\
\hline Control $\ddagger$ & $32.1 \pm 1.8$ & $31.7 \pm 10.3$ \\
Chronic & $15.8 \pm 2.7^{*}$ & $13.0 \pm 5.0^{*+}$ \\
\hline
\end{tabular}

${ }^{*}$ Differs from control vehicle $p<0.05, F^{\prime} s=24.0,32.4$.

$\dagger$ Differs from imipramine control $p<0.05, F^{*} s=20.0$.

\pm Respective tap water consumption for the four groups $=18 \pm 3$; $14 \pm 7 ; 15 \pm 7: 16 \pm 6 ;(F<1$. n.s. $)$.

TABLE 3

EFFECTS OF CHRONIC IMIPRAMINE HCI UPON WATER CONSUMPTION (IN ml) IN NORMAL AND CHRONICALI.Y STRESS RATS (ONE BOTTLE TEST)

\begin{tabular}{lll}
\hline & \multicolumn{3}{c}{ Drug } \\
$\begin{array}{l}\text { Stress } \\
\text { Condition }\end{array}$ & Vehicle & Imipramine \\
\hline Control $^{+}$ & $34.1 \pm 4.5$ & $26.1 \pm 2.0$ \\
Chronic & $28.3 \pm 6.6$ & $25.5 \pm 6.4^{*}$ \\
\hline
\end{tabular}

*Differs from control vehicle $p<0.05, \mathrm{~F}^{\prime} \mathrm{s}=8.1$.

tDiffers from chronic stress vehicle $p<0.05, F^{\prime} s=12.8,4.2$.

surprising that some degree of impairment in water consumption would be present given the ability of the rat to sense water as a separate taste modality [7].

The present findings argue that the depression model may share several similarities with clinical depression. As we have previously noted an appetitive deficit is well established as part of depression. The present findings therefore both confirm and extend the validity of the model.

\section{ACKNOWLEDGEMENTS}

Supported in part by grant MH31588 to Drs. B. J. Carroll (chief investigator) and $R$. J. Katz (co-investigator) from the National Institute of Mental Health. Dr. Katz is currently an Alfred P. Sloan research fellow in neuroscience. The expert editorial assistance of Anne Marie Skepenaitis and statistical consultation of R. C. Shea are acknowledged with gratitude. These experiments were carried out following extensive conversations with Kevin A. Roth and Michael Sibel. I am grateful for their useful comments and advice. 


\section{REFERENCES}

1. Bruning, J. L. and B. L. Kintz. Computational Handbook of Statistics, 2nd ed. Glenview, IL: Scott Foresman Co., 1977.

2. Katz, R. J. and S. Hersh. Amitriptyline and scopolamine in an animal model of depression. Neurosci. Biobehar. Rer. 5: 265281,1981 .

3. Katz, R. J., K. A. Roth, I. A. Mefford, B. J. Cartoll and J. Barchas. The chronically stressed rat-a novel animal of endogenomorphic depression. Proc. 3rd Int. Cong. Biol. Psychiat. Stockholm, Sweden, 1981.

4. Klein, D. Endogenomorphic depression, a conceptual and terminological revision. Archs gen. Psvchiat. 31: 447-454, 1974
5. Kraines, S. H. Manic depressive syndrome: A physiologic disease. Dis. nerv. Syst. 27: 3-19. 1966

6. Liljestrand, G. and Y. Zotterman. The water test in mammals Acta physiol. scand. 32: 291-303. 1954.

7. Pfaffmann, C. Gustatory nerve impulses in rat, cat, and rabbit. $J$. N'urophisiol. 18: 429-440, 1955

8. Roth, K. A. and R. J. Katz. Further studies on a novel animal model of depression therapeutic effects of a tricyclic antidepressant. Néurosci. Biobehav. Re'v. 5: 253-258, 1981. 